\title{
Three-dimensional printing in the preoperative planning of thoracoscopic pulmonary segmentectomy
}

\author{
Xiaojun Liu ${ }^{1, \#}$, Yandong Zhao ${ }^{1 \#}$, Yunpeng Xuan ${ }^{1 \#}$, Xinyan $\operatorname{Lan}^{2}$, Jun Zhao ${ }^{1,2}$, Xiaoquan $\operatorname{Lan}^{3}$, Bin Han $^{1}$, \\ Wenjie Jiao ${ }^{1}$ \\ ${ }^{1}$ Department of Thoracic Surgery, The Affiliated Hospital of Qingdao University, Qingdao 266003, China; ${ }^{2}$ Department of Thoracic Surgery, \\ ${ }^{3}$ Clinical Medical Transformation Center of Three-Dimensional Printing, Qingdao Chengyang District People's Hospital, Qingdao 266003, China \\ Contributions: (I) Conception and design: X Liu, Y Zhao, Y Xuan, W Jiao; (II) Administrative support: X Lan, X Lan, W Jiao; (III) Provision of study \\ materials or patients: All authors; (IV) Collection and assembly of data: X Liu, Y Zhao, Y Xuan, J Zhao, B Han; (V) Data analysis and interpretation: \\ X Liu, Y Zhao, Y Xuan, W Jiao; (VI) Manuscript writing: All authors; (VII) Final approval of manuscript: All authors. \\ \#These authors contributed equally to this work. \\ Correspondence to: Wenjie Jiao. Department of Thoracic Surgery, The Affiliated Hospital of Qingdao University, No. 16 Jiangsu Road, Shinan \\ District, Qingdao 266003, China. Email: jiaowj@qduhospital.cn.
}

Background: The purpose of this study is to explore whether $3 \mathrm{D}$ printing has a better clinical value for making a preoperative plan than three-dimensional computed tomography (3D-CT) in thoracoscopic pulmonary segmentectomy.

Methods: We collected a total of 124 patients' clinical data who underwent thoracoscopic pulmonary segmentectomy from October 2017 to August 2018. According to the preoperative examination, the patients were divided into three groups: general group, 3D-CT group, and 3D printing group. The clinical data of each group were analyzed and compared.

Results: Compared with the general group, intraoperative blood loss in 3D-CT group and 3D printing group decreased significantly $(\mathrm{P}<0.05)$. Operation time in $3 \mathrm{D}-\mathrm{CT}$ group and $3 \mathrm{D}$ printing group was significantly shorter than in the general group $(\mathrm{P}<0.05)$. Between $3 \mathrm{D}-\mathrm{CT}$ group and $3 \mathrm{D}$ printing group intraoperative blood loss and operation time had no significant differences $(\mathrm{P}>0.05)$. Postoperative chest tube duration and postoperative hospital stay had no significant differences between each group $\mathrm{P}>0.05)$. The incidence of postoperative hemoptysis in the general group occurred higher than in the 3D-CT group and $3 \mathrm{D}$ printing group, but the differences were not statistically significant $(\mathrm{P}>0.05)$. Postoperative complications of pneumonia, atelectasis, and pulmonary air leakage $(>6 \mathrm{~d})$ had no significant differences between each group $(\mathrm{P}>0.05)$.

Conclusions: 3D printing and 3D-CT for making a preoperative plan have an equivalent effect in thoracoscopic pulmonary segmentectomy for experienced surgeons. Preoperative simulations using 3D printing for the assessment of pulmonary vessel and bronchi branching patterns is beneficial for the safe and efficient performance of thoracoscopic pulmonary segmentectomy.

Keywords: Three-dimensional computed tomography (3D-CT); three-dimensional printing (3D printing); pulmonary segmentectomy

Submitted Aug 21, 2019. Accepted for publication Oct 29, 2019.

doi: $10.21037 /$ tlcr.2019.11.27

View this article at: http://dx.doi.org/10.21037/tlcr.2019.11.27 


\section{Introduction}

With the widespread use of chest computed tomography (CT) in physical examination, increasingly early-stage lung cancer has been found. For the treatment of earlystage lung cancer, many institutions have begun to perform pulmonary segmentectomy. Retrospective researches have indicated that for the patients with stage I non-small cell lung cancer (NSCLC) who underwent pulmonary segmentectomy or lobectomy, the disease-free survival and overall survival have no significant differences (1-3). It is believed that pulmonary segmentectomy will be performed increasingly to treat early-stage lung cancer in the future.

Compared with the pulmonary lobe, the anatomical structure of the lung segment is complex and prone to variation. Therefore, it is especially important to find techniques that can better display the anatomical structure of the pulmonary segment and guide pulmonary segmentectomy. Three-dimensional computed tomography (3D-CT) can well reconstruct the $3 \mathrm{D}$ images of vessels, bronchi, and tumor of the patient's diseased pulmonary lobe and now is widely used to make a preoperative plan. However, 3D-CT still shows 3D images on 2D screens, which still has great limitations in viewing the anatomy of pulmonary vessels and bronchi.

Three-dimensional printing (3D printing) is a new digital printing technology that can perfectly manufacture the physical model of human organs and present the anatomical structure of human organs perfectly. 3D printing has been proven to be beneficial in various fields of surgery (4-6). These 3D models can be especially useful for pre- and intraoperative surgical procedure planning. In this study, we will explore whether $3 \mathrm{D}$ printing has a better clinical value than $3 \mathrm{D}-\mathrm{CT}$ in thoracoscopic pulmonary segmentectomy.

\section{Methods}

\section{Patients choice}

We retrospectively analyzed the clinical data of patients who underwent thoracoscopic pulmonary segmentectomy in our institution from October 2017 to August 2018, excluding patients who were converted to lobectomy because of lymph node metastasis, and patients with severe pleural adhesion, the total number of patients was 124. Among them, 71 patients were reconstructed 3DCT images of pulmonary arteries, veins, and bronchi to make a preoperative plan, while the rest only made the preoperative plan through general enhanced chest CT.
By seeing 3D-CT reconstruction images, we found that the 3D-CT images of some patients (a total of 32 cases) were not conducive to observation because of the overlap of pulmonary arteries and veins. We applied 3D printing technology to convert these 3D-CT images into $3 \mathrm{D}$ printed models for preoperative observation and preoperative planning. Accordingly, we divided the patients into three groups: the general group, the 3D-CT group, and the $3 \mathrm{D}$ printing group. According to the NCCN guidelines and our clinical experience, the operative indications for pulmonary segmentectomy in our institution were as follow: (I) NSCLC in situ or minimally invasive NSCLC nodules with diameter $\leq 2 \mathrm{~cm}$; (II) ground glass density nodule component is $\geq 50 \%$ and nodal doubling time is $\geq 400$ days; (III) the nodule is in a deep position and difficult to locate; (IV) solitary lung metastatic tumor; (V) patient with poor pulmonary function or with other complications who is not eligible for lobectomy.

The Ethics Committee of the Affiliated Hospital of Qingdao University approved the study (approval number: QYFYKY 2018-10-11-2). Written informed consent was obtained from all patients before surgery.

\section{Preoperative 3D image reconstruction, 3D model printing and operative procedure}

(I) Preoperative 3D image reconstruction: $0.625-\mathrm{mm}$ data slices of the contrast-enhanced CT images was obtained using 64-channel multi-detector CT (MDCT), mimics (Materialise NV Technologielaan Leuven, Belgium) software was used in a personal computer to reconstruct $3 \mathrm{D}$ images of nodules, bronchi, and pulmonary vessels;

(II) $3 \mathrm{D}$ model printing: $0.625-\mathrm{mm}$ data slices of the contrast-enhanced CT images was obtained using 64-channel MDCT. 3D images of nodules, bronchi and pulmonary vessels were reconstructed by Mimics software, and then the 3D models were printed by Lite600HD 3D printer (Shanghai Union Technology CO., Ltd, Shanghai, China);

(III) Operative procedure: patients were administered general anesthesia with double-lumen ventilation and lay in the lateral decubitus position. The operation was performed with 2 or 3 incisions. A 10-mm camera port was placed in the seventh or eighth intercostal space at the midaxillary line and a 30 - or 40-mm incision in the fourth or fifth intercostal space at the anterior axillary line for the operating hole. If the operation is difficult, 
A $15 \mathrm{~mm}$ assist port was placed in the eighth or ninth intercostal space at the scapular line. According to the preoperative plan made by the $3 \mathrm{D}-\mathrm{CT}$ image or $3 \mathrm{D}$ printing model, the arteries and veins of the target segment were found carefully during the operation to avoid accidental injury (Figures 1,2). The "lung expansion-collapse method" was used to find segmental borders. The bronchus of target segment was clamped, and the lung was inflated to make sure the bronchus of target segment was correct, and then resected the target segmental bronchus and inflated the lung tissue completely to expand. Onelung ventilation of healthy side was kept for about 15 minutes, then in the diseased side, the targeted segment would still expend, and the other segments of the lung would collapse. According to the boundary between the inflatable target lung tissue and the collapsed lung tissue, the target segment was resected. All surgical margins of malignant nodules must be no less than $2 \mathrm{~cm}$, or at least equal to the diameter of the mass. Otherwise, combined pulmonary segmentectomy should be performed when necessary.

\section{Statistical analysis}

SPSS version 22.0 (IBM Corp., Armonk, NY, USA) was used to analyze the data. Continuous variables were reported as means $\pm \mathrm{SD}$ (standard deviation). Categorical variables were reported as frequency and percentage. Between groups, analyses of continuous variables were performed by the variance analysis, and the $\chi^{2}$ or Fisher's exact test were used to compare proportions in analyzing the frequency of occurrence of intraoperative and postoperative complications. A $\mathrm{P}$ value of $<0.05$ was considered statistically significant.

\section{Results}

The clinical characteristics of patients of each group are summarized in Table 1. There were no differences between each group. The segmentectomy position and number of cases are summarized in Table 2.

In Table 3, the study shows that compared with the general group, intraoperative blood loss in 3D-CT group and $3 \mathrm{D}$ printing group decreased significantly $(\mathrm{P}<0.05)$. Operation time in $3 \mathrm{D}-\mathrm{CT}$ group and $3 \mathrm{D}$ printing group was significantly shorter than in the general group, and the differences were statistically significant $(\mathrm{P}<0.05)$.
Postoperative chest tube duration and postoperative hospital stay had no significant differences between each group $(\mathrm{P}>0.05)$. Between $3 \mathrm{D}-\mathrm{CT}$ group and $3 \mathrm{D}$ printing group, the above variables had no significant differences; the differences were not statistically significant $(\mathrm{P}>0.05)$. Table 3 also records intraoperative and postoperative complications of each group. There were 5 conversions from thoracoscopic segmentectomy to thoracotomy or lobectomy in the general group, of which 4 patients had to convert to thoracotomy because of massive bleeding caused by intraoperative arterial injury, and one was forced to convert from trisegmentectomy of left upper lobe to left upper lobectomy, because of an anatomical variant mediastinal lingular branches of the pulmonary artery was injured during the operation. One case in the $3 \mathrm{D}-\mathrm{CT}$ group was converted to thoracotomy due to intraoperative vascular injury, and there was no conversion in the $3 \mathrm{D}$ printing group. The incidence of postoperative hemoptysis in the general group occurred higher than in the $3 \mathrm{D}-\mathrm{CT}$ group and $3 \mathrm{D}$ printing group, but the differences were not statistically significant $(P>0.05)$, we believed that the intersegmental veins were confirmed preoperatively through 3D-CT images and 3D printing models, and intraoperative protection was paid to avoid injury, and then the incidence of postoperative hemoptysis was reduced. Postoperative complications of pneumonia, atelectasis, and pulmonary air leakage ( $>6 \mathrm{~d})$ have no significant differences between each group $(\mathrm{P}>0.05)$.

\section{Discussion}

At present, lobectomy is still recognized as the standard operation for lung cancer treatment. Pulmonary segmentectomy for lung cancer treatment is still in controversy. It is mainly used for lung cancer patients with cardiopulmonary insufficiency who cannot tolerate pulmonary lobectomy because the pulmonary segmentectomy can maximally preserve healthy lung tissue to protect the patients' lung function, improve the quality of the patients' postoperative life (7). The application of chest CT in health examination makes more and more early-stage NSCLC being detected. Recently some retrospective studies have shown that in the treatment of stage I NSCLC, especially when the diameter of the tumor is less than $2 \mathrm{~cm}$, pulmonary segmentectomy and lobectomy have similar long-term effects. There were no significant differences between the two groups of NSCLC patients who underwent lobectomy or pulmonary segmentectomy 

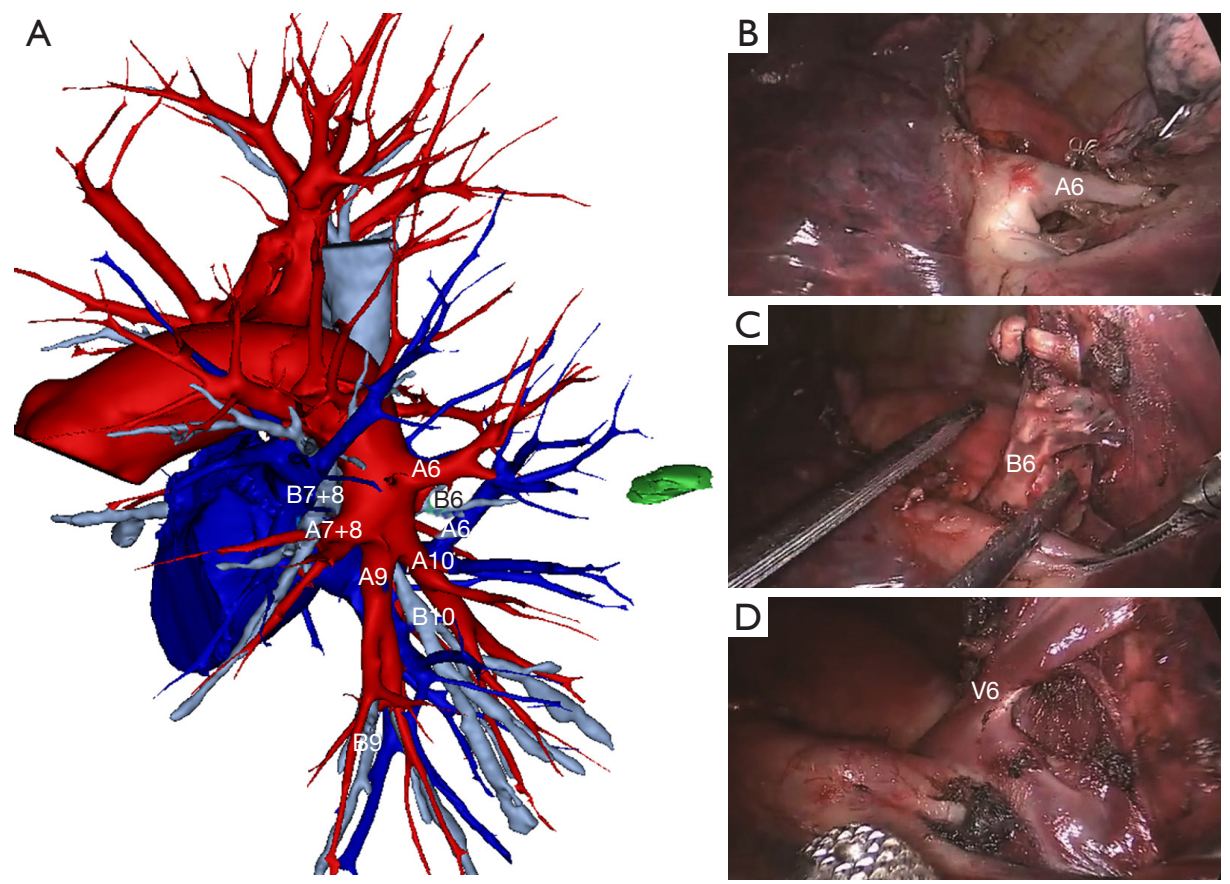

Figure 1 Photos from thoracoscopic LS6 segmentectomy. (A) Reconstructing 3D-CT image for LS6 segmentectomy; (B) pulmonary artery of LS6; (C) bronchus of LS6; (D) pulmonary vein of LS6.

in terms of the average days of hospitalization, incidence of complications, postoperative local recurrence rate, 5-year non-recurrence survival rate and 5-year overall survival rate (8-10). Therefore, more and more medical institutions perform pulmonary segmentectomy to treat early-stage NSCLC.

With the development of video-assisted technique, video-assisted thoracoscopic surgery (VATS) has become the main operative approach in Department of thoracic surgery. Its operative effect has been confirmed by researches and is superior to thoracotomy $(11,12)$. Compared with thoracoscopic lobectomy, thoracoscopic pulmonary segmentectomy requires a higher level of surgery skills and deeper understanding of the anatomy of lung segment. The difficulty of the operation is to accurately determine the arteries, veins, and bronchi of the affected pulmonary segment and the boundary of the segment. Compared with pulmonary lobe, there are more anatomical variations in the pulmonary segment. If the anatomical structure of pulmonary segment cannot be fully distinguished before surgery, intraoperative injuries are likely to be caused. The study has shown that intraoperative vascular injury due to vascular anatomical variations in the pulmonary segment is an important factor in the conversion of thoracoscopic pulmonary segmentectomy to thoracotomy or lobectomy (13). So fully understanding individual anatomical variations of pulmonary arteries, veins and bronchi are important for operative safety, especially when performing anatomical pulmonary segmentectomy. It is considered that preoperative modeling is one of the most important tools to ensure the successful performance of thoracoscopic pulmonary segmentectomy (13). In our study, 5 patients in the general group were converted to thoracotomy or lobectomy due to vascular injury, while only 1 patient in the 3D-CT group was converted to thoracotomy due to vascular injury, and there were no conversions in the $3 \mathrm{D}$ printing group. Postoperative hemoptysis is a serious and common complication after pulmonary segmentectomy. Compared with the general group, the incidence of postoperative hemoptysis in the $3 \mathrm{D}$-CT group and the $3 \mathrm{D}$-printing group was lower. These all prove the importance of preoperative modeling for thoracoscopic pulmonary segmentectomy.

$3 \mathrm{D}-\mathrm{CT}$ is the most widely used preoperative modeling technology in clinical and has been widely used in various clinical disciplines. In thoracic surgery, 3D-CT was used to reconstruct 3D images of vessels, bronchi, and tumors of the target pulmonary lobe. By seeing 3D images, the 

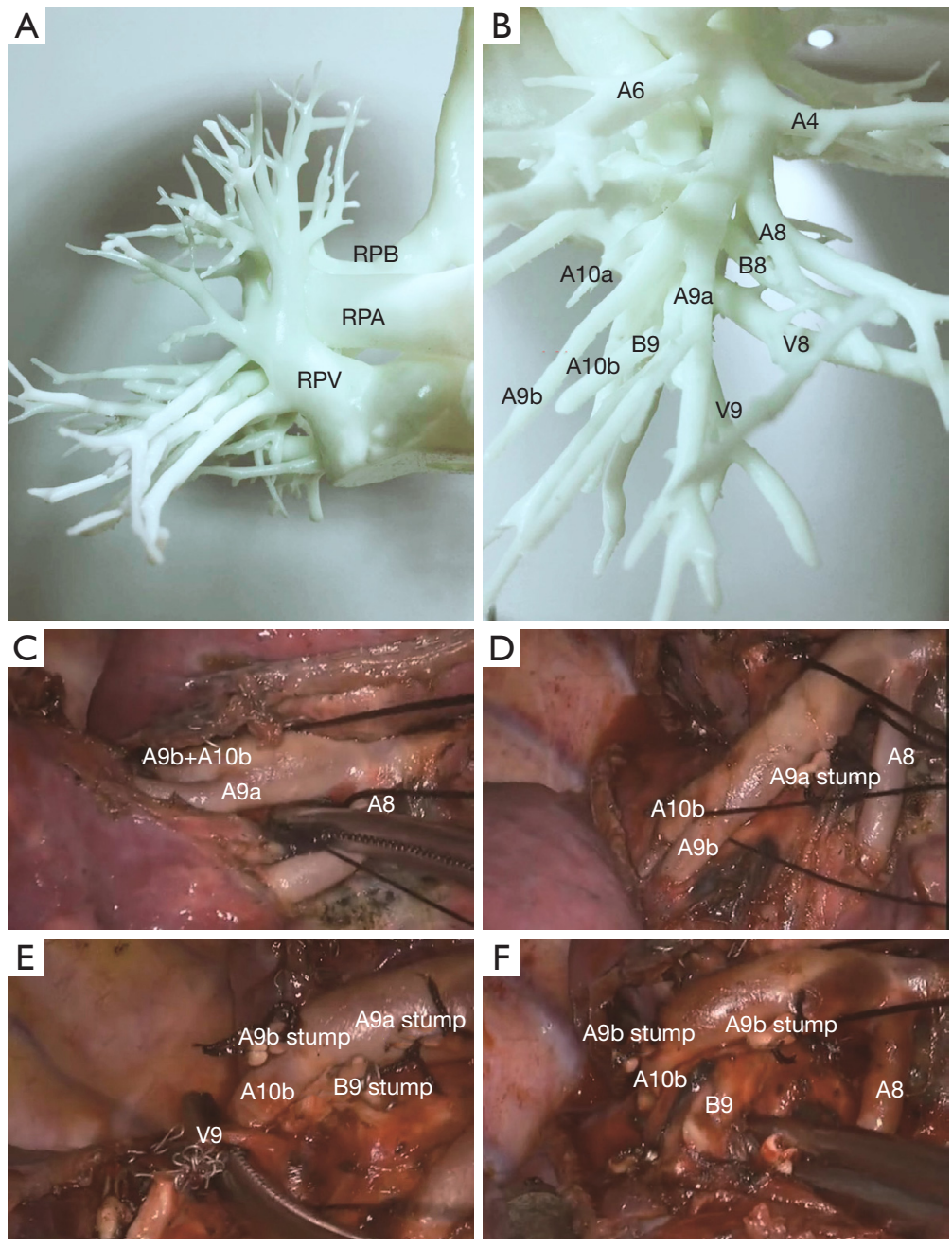

Figure 2 Photos from thoracoscopic RS9 segmentectomy. (A) 3D printed model of vessels and bronchi of the right lobe; (B) 3D printed model of arteries, veins, and bronchi of the right low lobe; (C) pulmonary arteries of RS9; (D) pulmonary arteries of RS9; (E) pulmonary vein of RS9; (F) bronchus of RS9. RPA, right pulmonary artery; RPB, right pulmonary bronchus; RPV, right pulmonary vein.

distribution and variation of the bronchi and vessels in the target pulmonary lobe were clarified, and preoperative plans were made to prevent intraoperative injuries. A lot of studies have been made about the use of 3D-CT in pulmonary segmentectomy. Masaru Hagiwara's study showed that patients with thoracoscopic pulmonary segmentectomy or lobectomy undergoing preoperative $3 \mathrm{D}$ imaging tended to have lower incidence of postoperative complications and have significantly shorter operative time. It concluded that preoperative simulations using $3 \mathrm{D}$ CT angiography for the assessment of pulmonary vessel branching patterns appear to be beneficial for the safe and efficient performance of thoracoscopic pulmonary segmentectomy and lobectomy (14). Studies by Oizumi had shown that $3 \mathrm{D}-\mathrm{CT}$ could effectively guide the thoracoscopic pulmonary segmentectomy and ensure the safety of the operation, especially when difficult pulmonary segmentectomy was performed (15). Saji proposed virtual segmentectomy. Lung imaging model was set up by $3 \mathrm{D}-\mathrm{CT}$, and the arterial and bronchial branches of the target pulmonary segments were determined before surgery. The boundary of the target lung segment was determined by observing the bronchial branches ventilation area of the target lung segment, and the preoperative estimated surgical margin was sufficient to enable the surgeon to accurately complete anatomic pulmonary 
Table 1 Clinical characteristics of patients of each group

\begin{tabular}{|c|c|c|c|c|}
\hline Characteristics & General group $(n=53)$ & 3D-CT group $(n=39)$ & $3 D$ printing group $(n=32)$ & $P$ \\
\hline \multicolumn{5}{|l|}{ Sex, n (\%) } \\
\hline Male & $19(36)$ & $13(33)$ & $13(41)$ & \multirow[t]{2}{*}{0.814} \\
\hline Female & $34(64)$ & $26(67)$ & $19(59)$ & \\
\hline \multicolumn{5}{|l|}{ Pathology, n (\%) } \\
\hline NSCLC & $45(84.9)$ & $34(87.2)$ & $27(84.4)$ & \multirow[t]{3}{*}{0.954} \\
\hline Metastatic tumor & $2(3.8)$ & $2(5.1)$ & $1(3.1)$ & \\
\hline Benign & $6(11.3)$ & $3(7.7)$ & $4(12.5)$ & \\
\hline Diabetes mellitus & $5(9.4)$ & $3(7.7)$ & $3(9.4)$ & 0.951 \\
\hline COPD & $6(11.3)$ & $4(10.3)$ & $4(12.5)$ & 0.957 \\
\hline Heart diseases & $3(5.7)$ & $3(7.7)$ & $2(6.3)$ & 0.809 \\
\hline
\end{tabular}

NSCLC, non-small cell lung cancer; COPD, chronic obstructive pulmonary disease.

segmentectomy and achieve radical resection (16). Our study showed that compared with the general group, the operation time in the 3D-CT group were shorter, and intraoperative blood loss in the $3 \mathrm{D}-\mathrm{CT}$ group was less. 3D-CT can ensure the faster and safer completion of thoracoscopic pulmonary segmentectomy.

$3 \mathrm{D}$ printing is a modern technology in recent years. Compared with $3 \mathrm{D}-\mathrm{CT}$ showing $3 \mathrm{D}$ images on a $2 \mathrm{D}$ screen, 3D printed model can be seen from any angle in the hands of the operators. They can more clearly visualize and experience in a tactile manner the three-dimensional relationships of each structure to another in real scale.3D printing was first applied to orthopedics and plastic surgery department, and now it is applied to various clinical disciplines. The application of $3 \mathrm{D}$ printing technology in thoracic surgery is rare, and still in its infancy, there were only a few cases reported its use in thoracoscopic pulmonary segmentectomy, and no systematic study has been made. Akiba et al. reported that $3 \mathrm{D}$ printing was used to make preoperative plan for thoracoscopic pulmonary segmentectomy of 2 patients with dysplasia of pulmonary fissure and achieved good results (17). From October 2017, our institution began to use 3D printing technology for some patients who needed to perform thoracoscopic pulmonary segmentectomy to make preoperative plan. The
1:1 3D model of arteries, veins, and bronchi of the affected lung were printed to guide thoracoscopic pulmonary segmentectomy. Our study showed that compared with the general group, the operation time in the $3 \mathrm{D}$ printing group were shorter, and intraoperative blood loss in the 3D printing group was less, but there were no differences between $3 \mathrm{D}$ printing group and $3 \mathrm{D}-\mathrm{CT}$ group. Preoperative determination of the arteries, veins, and bronchi of the target pulmonary segments could shorten the intraoperative identification time, reduce excessive tissue separation, avoid intraoperative accidental injury, and reduce intraoperative blood loss. The operations of the patients in our study were performed by experienced operators, who performed about 300 cases of thoracoscopic lobectomy and 100 cases of thoracoscopic pulmonary segmentectomy annually. Therefore, the surgeons have extremely high surgical skill and have a good knowledge of the 3D distribution of pulmonary arteries, veins, and bronchi. So, we believe that the $3 \mathrm{D}-\mathrm{CT}$ image is enough for experienced surgeons to see the distribution and variation of pulmonary arteries, veins, and bronchi before surgery. 3D printing cannot more significantly improve the surgical effect of thoracoscopic pulmonary segmentectomy performed by experienced surgeons than $3 \mathrm{D}-\mathrm{CT}$.

Although our study showed that the 3D printing had no 
Table 2 Segmentectomy position and number of cases of each group

\begin{tabular}{|c|c|c|c|}
\hline Segmentectomy position & General group $(n=53)$ & 3D-CT group $(n=39)$ & $3 \mathrm{D}$ printing group $(\mathrm{n}=32)$ \\
\hline $\mathrm{LS} 1+2+3$ & 5 & 2 & 4 \\
\hline LS4+5 & 5 & 3 & - \\
\hline LS6 & 9 & 4 & 1 \\
\hline LS7+8 & 1 & 2 & 2 \\
\hline $\mathrm{RS} 1$ & 4 & 2 & 3 \\
\hline $\mathrm{RS} 2$ & 4 & 3 & 4 \\
\hline RS3 & 5 & 3 & 3 \\
\hline $\mathrm{RS} 7+8+9+10$ & 1 & 1 & - \\
\hline $\mathrm{RS} 7+8$ & 1 & 2 & 1 \\
\hline RS7 & - & 2 & 2 \\
\hline RS9 & - & 1 & 1 \\
\hline
\end{tabular}

Table 3 Intraoperative and postoperative data of each group

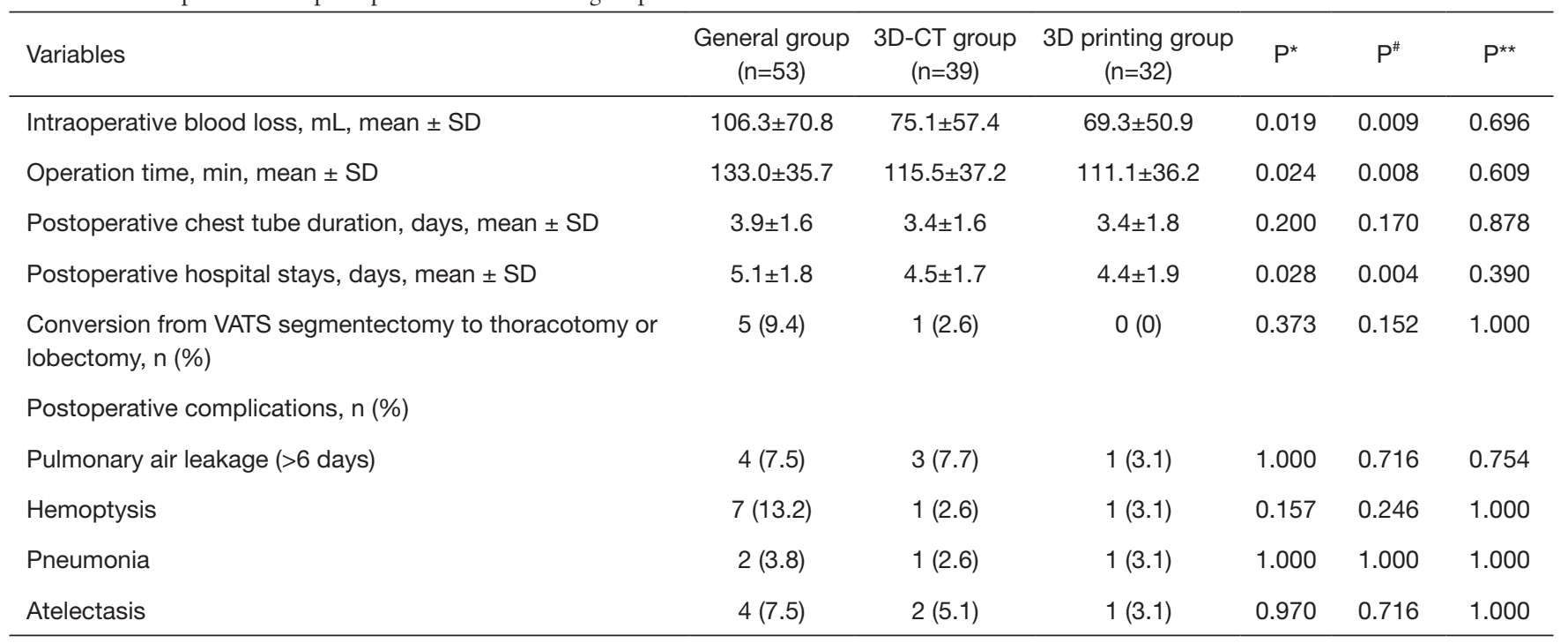

*, general group and 3D-CT group; ", general group, and 3D printing group; **, 3D-CT group and 3D printing group.

significant improvement on the thoracoscopic pulmonary segmentectomy for experienced operators, we found that the $3 \mathrm{D}$ printed models were of immense help to the clinical learning of thoracic surgery residents. For the 3D printing group and 3D-CT group, we asked the residents to make preoperative plan according to $3 \mathrm{D}$ printed models or $3 \mathrm{D}$ CT images (including identifying the pulmonary segment where the tumor located, and the bronchi, arteries, veins of the target pulmonary segment). Viewing $3 \mathrm{D}$ printed models could help residents to make preoperative plans 
faster and better, while 3D-CT images were difficult for residents with immature pulmonary anatomy and immature stereoscopic thinking to understand. 3D printing models can shorten the learning curve of thoracic surgery residents. This is consistent with relevant literature reports $(18,19)$. Such models are useful for educational purposes in resident physician training because they increase $3 \mathrm{D}$ perception and add tactile feedback to the trainee. $3 \mathrm{D}$ printing models can also promote doctor-patient communication. Before surgery, we respectively used $3 \mathrm{D}$ printed models and $3 \mathrm{D}$ CT images to explain the operation plans to the patients' family members. The family members of patients with $3 \mathrm{D}$ printed models could understand the operation intention and accept the operation plan better and faster.

In conclusion, this study shows that $3 \mathrm{D}-\mathrm{CT}$ and $3 \mathrm{D}$ printing for making preoperative plan have an equivalent effect in thoracoscopic pulmonary segmentectomy for experienced surgeons. Preoperative simulations using $3 \mathrm{D}$ printing for the assessment of pulmonary vessels and bronchi branching patterns is beneficial for the safe and efficient performance of thoracoscopic pulmonary segmentectomy. Although it takes longer to create 3D printed model and cost more, $3 \mathrm{D}$ printing is an especially useful tool for thoracic surgery.

\section{Acknowledgments}

None.

\section{Footnote}

Conflicts of Interest: The authors have no conflicts of interest to declare.

Ethical Statement: The authors are accountable for all aspects of the work in ensuring that questions related to the accuracy or integrity of any part of the work are appropriately investigated and resolved. The Ethics Committee of the Affiliated Hospital of Qingdao University approved the study (approval number: QYFYKY 201810-11-2). Written informed consent was obtained from all patients before surgery.

\section{References}

1. Ren M, Meng Q, Zhou W, et al. Comparison of shortterm effect of thoracoscopic segmentectomy and thoracoscopic lobectomy for the solitary pulmonary nodule and early-stage lung cancer. Onco Targets Ther 2014;7:1343-7.

2. Zhong C, Fang W, Mao T, et al. Comparison of Thoracoscopic Segmentectomy and Thoracoscopic Lobectomy for Small-Sized Stage IA Lung Cancer. Ann Thorac Surg 2012;94:362-7.

3. Hwang Y, Kang CH, Kim HS, et al. Comparison of thoracoscopic segmentectomy and thoracoscopic lobectomy on the patients with non-small cell lung cancer: a propensity score matching study. Eur J Cardiothorac Surg 2015;48:273-8.

4. Xiang N, Fang C, Fan Y, et al. Application of liver three-dimensional printing in hepatectomy for complex massive hepatocarcinoma with rare variations of portal vein: preliminary experience. Int J Clin Exp Med 2015;8:18873-8.

5. Choi JW, Kim N. Clinical Application of ThreeDimensional Printing Technology in Craniofacial Plastic Surgery. Arch Plast Surg 2015;42:267-77.

6. McGovern E, Kelleher E, Snow A, et al. Clinical application of three-dimensional printing to the management of complex univentricular hearts with abnormal systemic or pulmonary venous drainage. Cardiol Young 2017;27:1248-56.

7. Kim SJ, Lee YJ, Park JS, et al. Changes in Pulmonary Function in Lung Cancer Patients After Video-Assisted Thoracic Surgery. Ann Thorac Surg 2015;99:210-7.

8. Fan J, Wang L, Jiang GN, et al. Sublobectomy Versus Lobectomy for Stage I Non-Small-Cell Lung Cancer, A Meta-Analysis of Published Studies. Ann Surg Oncol 2012;19:661-8.

9. Nakamura H, Taniguchi Y, Miwa K, et al. Comparison of the Surgical Outcomes of Thoracoscopic Lobectomy, Segmentectomy, and Wedge Resection for Clinical Stage I Non-Small Cell Lung Cancer. Thorac Cardiovasc Surg 2011;59:137-41.

10. Schuchert MJ, Abbas G, Awais O, et al. Anatomic Segmentectomy for the Solitary Pulmonary Nodule and Early-Stage Lung Cancer. Ann Thorac Surg 2012;93:1780-5; discussion 1786-7.

11. Cao C, Zhu ZH, Yan TD, et al. Video-assisted thoracic surgery versus open thoracotomy for non-small-cell lung cancer: a propensity score analysis based on a multi-institutional registry. Eur J Cardiothorac Surg 2013;44:849-54.

12. Pan TW, Wu B, Xu ZF, et al. Video-assisted Thoracic Surgery Versus Thoracotomy for Non-small-cell Lung Cancer. Asian Pac J Cancer Prev 2012;13:447-50. 
13. Gossot D, Lutz JA, Grigoroiu M, et al. Unplanned Procedures During Thoracoscopic Segmentectomies. Ann Thorac Surg 2017;104:1710-7.

14. Hagiwara M, Shimada Y, Kato Y, et al. High-quality 3-dimensional image simulation for pulmonary lobectomy and segmentectomy: results of preoperative assessment of pulmonary vessels and short-term surgical outcomes in consecutive patients undergoing video-assisted thoracic surgery†. Eur J Cardiothorac Surg 2014;46:e120-6.

15. Oizumi H, Kanauchi N, Kato H, et al. Anatomic thoracoscopic pulmonary segmentectomy under 3-dimensional multidetector computed tomography simulation: A report of 52 consecutive cases. J Thorac Cardiovasc Surg 2011;141:678-82.

16. Saji H, Inoue T, Kato Y, et al. Virtual segmentectomy

Cite this article as: Liu X, Zhao Y, Xuan Y, Lan X, Zhao J, Lan X, Han B, Jiao W. Three-dimensional printing in the preoperative planning of thoracoscopic pulmonary segmentectomy. Transl Lung Cancer Res 2019;8(6):929-937. doi: $10.21037 /$ tlcr.2019.11.27 based on high-quality three-dimensional lung modelling from computed tomography images. Interact Cardiovasc Thorac Surg 2013;17:227-32.

17. Akiba T, Nakada T, Inagaki T. Simulation of the Fissureless Technique for Thoracoscopic Segmentectomy Using Rapid Prototyping. Ann Thorac Cardiovasc Surg 2015;21:84-86.

18. Zheng YX, Yu DF, Zhao JG, et al. 3D Printout Models vs 3D-Rendered Images: Which Is Better for Preoperative Planning? J Surg Educ 2016;73:518-23.

19. Sakaguchi M, Maebayashi T, Aizawa T, et al. Synchronous organizing pneumonia after sequential three-dimensional radiotherapy for three lung metastases from hepatocellular carcinoma: a case report. Transl Cancer Res 2018;7:450-5. 